Pacific Journal of Mathematics

UNBOUNDED MULTIPLIERS ON COMMUTATIVE BANACH JLGEDRS 


\title{
UNBOUNDED MULTIPLIERS ON COMMUTATIVE BANACH ALGEBRAS
}

\section{JAMES WOOD}

\begin{abstract}
In this paper the notion of an unbounded multiplier on a commutative Banach algebra is introduced. It is proven that, as in the case of bounded multipliers, unbounded multipliers also have Gelfand transforms. Some of the properties of these transforms are then developed. The final result of the paper is a new characterization of the bounded multipliers on $A$ where $A$ is a Banach algebra of the type described below.
\end{abstract}

1. Introduction. In general, by a multiplier on a commutative Banach algebra $A$ one means a bounded linear operator $T: A \rightarrow$ $A$ such that $T(x y)=x T(y)$ for all $x, y \in A$. There is an extensive literature on the subject. One can consult, for example, [2] and [3]. There does not seem to be, however, a systematic treatment of unbounded multipliers although such multipliers occur quite naturally. For example, consider the Banach algebra $L^{1}(-\infty, \infty)$ with the convolution product and define $T$ by $T(f)=f^{\prime}$, where the domain $\mathscr{D}(T)$ of $T$ is the set $\left\{f \mid f \in L^{1}(-\infty, \infty)\right.$ and $f$ is absolutely continuous\}. It is easy to check that $T(f g)=f T(g)$ almost everywhere for all $f \in L^{1}(-\infty, \infty)$ and for all $g \in \mathscr{D}(T)$. However, $T$ is not bounded. (Although it is closed.)

As another example, take the Banach algebra $C_{0}(-\infty, \infty)$ of all complex valued continuous functions on the real line which vanish at infinity with $T$ defined by $T(f)(x)=x f(x)$. The domain $\mathscr{D}(T)$ can be taken to be the set of all functions in $C_{0}(-\infty, \infty)$ with compact support. Finally, let $\left\{T_{t} \mid t \geqq 0\right\}$ be a semi-group of class $C_{0}$ of bounded multipliers on the Banach algebra $A$. Then the infinitesimal generator $T_{0}$ of $\left\{T_{t} \mid t \geqq 0\right\}$ is an (in general) unbounded multiplier, for if $x \in \mathscr{D}\left(T_{0}\right)$ and $y \in A$ then

$$
\begin{aligned}
T_{0}(x y) & =\lim _{h \downarrow 0} \frac{1}{h}[T(x y)-x y] \\
& =y \lim _{h \downarrow 0} \frac{1}{h}[T(x)-x]=y T_{0}(x) .
\end{aligned}
$$

It is the purpose of this present paper to study some of the properties of unbounded multipliers in the general setting of a commutative Banach algebra. In particular, henceforth $A$ always denotes a regular, commutative semi-simple Banach algebra. We also assume for the rest of the paper that $A$ has a bounded 
approximate identity $\left\{e_{\alpha}\right\}$ and that the Gelfand transform of each $\left\{e_{\alpha}\right\}$ has compact support. By $\Delta(A)$ we shall mean the maximal ideal space of $A$ with the Gelfand topology and the symbol $\hat{x}$ denotes the Gelfand transform of $x \in A$.

\section{Definitions and general properties.}

Definition 2.1. Let $A$ be a Banach algebra. The function $T$ defined on the dense ideal $\mathscr{D}(T) \subseteq A$ is called a multiplier of $A$ if and only if for all $x \in \mathscr{D}(T)$ and all $y \in A, T(x y)=y T(x)$.

The first theorem shows that, as in the bounded case, every multiplier has a Gelfand transform.

Theorem 2.1. Given a multiplier $T$ on $A$ there is a unique complex valued continuous function $T^{\wedge}$ on $\Delta(A)$ such that for all $\tau \in \Delta(A)$ and all $x \in \mathscr{D}(T), T(x)^{\wedge}(\tau)=T^{\wedge}(\tau) x^{\wedge}(\tau)$.

Proof. First of all observe that for each $\tau \in \Delta(A)$ there is an $x \in \mathscr{D}(T)$ with $x^{\wedge}(\tau) \neq 0$. For otherwise, if for some $\tau_{0}, x^{\wedge}\left(\tau_{0}\right)=0$ for all $x \in \mathscr{D}(T)$, then $\mathscr{D}(T)$ is contained in some maximal ideal which is a contradiction. Now if $\tau_{0} \in \Delta(A)$, choose $x \in \mathscr{D}(T)$ so that $x^{\wedge}\left(\tau_{0}\right) \neq 0$ and define $T^{\wedge}\left(\tau_{0}\right)$ by $T^{\wedge}\left(\tau_{0}\right)=T(x)^{\wedge}\left(\tau_{0}\right) / x^{\wedge}\left(\tau_{0}\right)$. The function $T^{\wedge}$ is independent of $x$, for if $y \in \mathscr{D}(T)$ and $y^{\wedge}\left(\tau_{0}\right) \neq 0$, then from $T(x y)=x T(y)=y T(x)$ follows $x^{\wedge}(\tau) T(y)^{\wedge}(\tau)=y^{\wedge}(\tau) T(x)^{\wedge}(\tau)$ for all $\tau \in \Delta(A)$, so that $T(y)^{\wedge}\left(\tau_{0}\right) / y^{\wedge}\left(\tau_{0}\right)=T(x)^{\wedge}\left(\tau_{0}\right) / x^{\wedge}\left(\tau_{0}\right)$. It is clear that $T^{\wedge}$ is continuous. To show uniqueness suppose there is a continuous function $\phi$ on $\Delta(A)$ such that $T(x)^{\wedge}(\tau)=\phi(\tau) x^{\wedge}(\tau)$ for all $x \in \mathscr{D}(T)$ and all $\tau \in \Delta(A)$. Then $T^{\wedge}(\tau) x^{\wedge}(\tau)=\phi(\tau) x^{\wedge}(\tau)$ for all $x \in \mathscr{D}(T)$ and $\tau \in \Delta(A)$ and since for each $\tau$ we can find an $x$ such that $x^{\wedge}(\tau) \neq 0$, it follows that $T^{\wedge}=\phi$.

Corollary 2.1. A multiplier is linear on its domain.

Proof. Suppose $x, y \in \mathscr{D}(T)$ and let $z=\alpha x+\beta y$ where $\alpha$ and $\beta$ are complex numbers. Then from $z^{\wedge}(\tau)=\alpha x^{\wedge}(\tau)+\beta y^{\wedge}(\tau)$ we get successively

$$
\begin{aligned}
& T^{\wedge}(\tau) z^{\wedge}(\tau)=\alpha T^{\wedge}(\tau) x^{\wedge}(\tau)+\beta T^{\wedge}(\tau) y^{\wedge}(\tau) \\
& T(z)^{\wedge}(\tau)=\alpha T(x)^{\wedge}(\tau)+\beta T(y)^{\wedge}(\tau) \\
& T(z)^{\wedge}(\tau)=(\alpha T(x)+\beta T(y))^{\wedge}(\tau) \\
& T(z)=\alpha T(x)+\beta T(y),
\end{aligned}
$$

the last equation following from the semi-simplicity of the algebra. 
THEOREM 2.2. Every multiplier is closable.

Proof. Let $T$ be a multiplier and assume $\left\{x_{n}\right\}$ is a sequence in $\mathscr{D}(T)$ with $\lim x_{n}=0$ and $\lim T\left(x_{n}\right)=y$. Since $T\left(x_{n}\right) \rightarrow y, T^{\wedge}(\tau) x_{n}{ }^{\wedge}(\tau) \rightarrow$ $y^{\wedge}(\tau)$. But $T^{\wedge}(\tau) x_{n}{ }^{\wedge}(\tau) \rightarrow 0$ also and thus $y^{\wedge}(\tau)=0$ for all $\tau \in \Delta(A)$ so that $y=0$.

THEOREM 2.3. If $S$ and $T$ are multipliers such that $S^{\wedge}(\tau)=$ $T^{\wedge}(\tau)$ for all $\tau$ and in addition $S$ and $T$ are closed then $S=T$.

Proof. The algebra has a bounded approximate identity $\left\{e_{\alpha}\right\}$. Now for each $\alpha$ and any $\varepsilon>0$ there is an $e_{\alpha}^{\prime} \in \mathscr{D}(S)$ such that $\left\|e_{\alpha}-e_{\alpha}^{\prime}\right\|<\varepsilon$. Thus $\left\{e_{\alpha}^{\prime}\right\}$ is an approximate identity in $\mathscr{D}(S)$. Similarly we can construct an approximate identity $e_{\alpha}^{\prime \prime}$ in $\mathscr{D}(T)$. Now letting $f_{\alpha}=e_{\alpha}^{\prime} e_{\alpha}^{\prime \prime}$, we have that $f_{\alpha} \in \mathscr{D}(S) \cap \mathscr{D}(T)$ and since $S^{\wedge}(\tau)=T^{\wedge}(\tau)$ we get $S^{\wedge}(\tau) e_{\alpha}^{\prime}(\tau) e_{\alpha}^{\prime \prime}(\tau)=T^{\wedge}(\tau) e_{\alpha}^{\prime}(\tau) e_{\alpha}^{\prime \prime}(\tau)$ so that $S\left(f_{\alpha}\right)=$ $T\left(f_{\alpha}\right)$. Now let $f \in \mathscr{D}(S)$. Then $f f_{\alpha} \rightarrow f$ and $S\left(f f_{\alpha}\right)=f_{\alpha} S(f) \rightarrow S(f)$. But $S\left(f f_{\alpha}\right)=f S\left(f_{\alpha}\right)=f T\left(f_{\alpha}\right)$ so that $T\left(f f_{\alpha}\right) \rightarrow S(f)$ also. Therefore, since $T$ is closed, $f \in \mathscr{D}(T)$ and $T(f)=S(f)$. By reversing the roles of $S$ and $T$ we get the assertion of the theorem.

COROLlaRy 2.2. If $S$ and $T$ are multipliers such that $S^{\wedge}=T^{\wedge}$, then $S$ and $T$ have the same closure.

Proof. If $S_{0}$ and $T_{0}$ denote the closures of $S$ and $T$ respectively, then $S_{0}^{\wedge}=S^{\wedge}$ and $T_{0}^{\wedge}=T^{\wedge}$. Therefore $S_{0}^{\wedge}=T_{0}^{\wedge}$ so that by the previous theorem $S_{0}=T_{0}$.

The previous theorem and corollary provide us in an obvious way with a means to define an equivalence relation on the set of multipliers. We say that $S$ is equivalent to $T, S \sim T$, if and only if $S^{\wedge}=T^{\wedge}$. Thus the set of multipliers can be split up into equivalence classes such that all multipliers in the same class have the same closure and the same transform.

The next theorem concerns inverses of multipliers.

THeOREM 2.4. Let $T$ be a multiplier. Then $T^{-1}$ exists and is a multiplier if and only if $T^{\wedge}$ never vanishes.

Proof. Assume $T^{-1}$ exists as a multiplier. Then for each $x \in \mathscr{D}\left(T^{-1}\right)$ there is a $z \in \mathscr{D}(T)$ such that $T^{\wedge}(\tau) z^{\wedge}(\tau)=x^{\wedge}(\tau)$. If $T^{\wedge}\left(\tau_{0}\right)=0$ for some $\tau_{0}$ then $x^{\wedge}\left(\tau_{0}\right)=0$ for all $x \in \mathscr{D}\left(T^{-1}\right)$ which contradictics the fact that $\mathscr{D}\left(T^{-1}\right)$ is dense. On the other hand assume that $T^{\wedge}$ never vanishes. Then the set $I=\{y \in A \mid x \in \mathscr{D}(T)$ with $\left.T^{\wedge}(\tau) x^{\wedge}(\tau)=y^{\wedge}(\tau)\right\}$ is an ideal in $A$. If $I$ is not dense, then its 
closure is a closed proper ideal and hence contained in a maximal ideal. Therefore, there is a $\tau_{0}$ such that $y^{\wedge}\left(\tau_{0}\right)=0$ for all $y \in I$ and this in turn implies $T^{\wedge}\left(\tau_{0}\right) x^{\wedge}\left(\tau_{0}\right)=0$ for all $x \in \mathscr{D}(T)$. Thus $x^{\wedge}\left(\tau_{0}\right)=0$ for all $x \in \mathscr{D}(T)$ which is a contradiction.

It is possible to characterize those functions on $\Delta(A)$ which are transforms of multipliers. This is done in the following theorem.

THEOREM 2.5. In order that $T^{\wedge}$ be the transform of a multiplier it is necessary and sufficient that $T^{\wedge}$ belong locally to $A^{\wedge}$ at each point of $\Delta(A)$.

Proof. If $T^{\wedge}$ belongs locally to $A^{\wedge}$ at each point of $\Delta(A)$, then $T^{\wedge} e_{\alpha} \wedge$ belongs locally to $A^{\wedge}$ at each point $\Delta(A) \cup\{\infty\}$. By a theorem due to F. Birtel, ([1], p. 818), this implies that $T^{\wedge} e_{\alpha}{ }^{\wedge}$ belongs to $A^{\wedge}$. Therefore, for any $x \in A, x^{\wedge} T^{\wedge} e_{\alpha}^{\wedge}$ belongs to $A^{\wedge}$ and we define $T\left(x e_{\alpha}\right)=y_{\alpha}$ where $y_{\alpha}{ }^{\wedge}=x^{\wedge} T^{\wedge} e_{\alpha}$. If we then let $\mathscr{D}(T)$ be $\left\{x e_{\alpha} \mid x \in\right.$ $A\}$, we have $T\left(z x e_{\alpha}\right)=y_{\alpha}$ where $y_{\alpha} \hat{\wedge}=z^{\wedge} x^{\wedge} T^{\wedge} e_{\alpha} \hat{}$. But $x^{\wedge} T^{\wedge} e_{\alpha}^{\wedge}=$ $T\left(x e_{\alpha}\right)^{\wedge}$ and so $y_{\alpha}=z T\left(x e_{\alpha}\right)$. Thus $T$ determines a multiplier. Conversely, if $T^{\wedge}$ is the transform of a multiplier and $p \in \Delta(A)$, there exists an $x \in \mathscr{D}(T)$ such that $x^{\wedge}(\tau) \neq 0$ on some compact neighborhood $V$ of $p$. There is then a $y \in A$ such that $y^{\wedge}(\tau)=1 / x^{\wedge}(\tau)$ for all $\tau \in V$ so that $x^{\wedge}(\tau) y^{\wedge}(\tau)=1$ on $V$. But $x y \in \mathscr{D}(T)$, so that if we let $z=T(x y)$, then $z^{\wedge}(\tau)=T^{\wedge}(\tau) x^{\wedge}(\tau) y^{\wedge}(\tau)$ for all $\tau \in \Delta(A)$ and $T^{\wedge}$ belongs locally to $A^{\wedge}$ at $p$.

COROLLARY 2.3. If $T^{\wedge}$ is the transform of a multiplier, then $T^{\wedge}$ is the transform of a bounded multiplier if and only if $|p(T(x))| \mid$ $\|p x\| \leqq M$ for all $p \in A^{*}$ and all $x \in \mathscr{D}(T)$. (Here $A^{*}$ is the dual of $A$ and $p x$ is the functional defined on $A$ by $p x(y)=p(x y)$ for all $y \in A$.)

Proof. The proof follows immediately by combining the above theorem and the following theorem proved in [4].

THeOREM. Let $\hat{F}$ be a complex valued function on $\Delta(A)$. In order that $\hat{F}$ determine a bounded multiplier of $A$, it is necessary and sufficient that $\hat{F}$ belongs locally to $\Delta(A)$ at each point of $\Delta(A)$ and that $|p(F(x))| /\|p x\| \leqq M$, for all $p \in A^{*}$ and for all $x \in A_{F} . \quad\left(A_{F}\right.$ is the set $\{x \mid \hat{F} \hat{x}=\hat{y}$ for some $y \in A$.)

3. Further results. In Theorem 2.5 we gave a necessary and sufficient condition that a function on $\Delta(A)$ be the transform of a multiplier. The next theorem gives a different necessary and sufficient condition that a function be the transform of a multiplier. 
This identity, as it turns out, allows us to characterize those functions which are transforms of bounded multipliers on the Banach algebra $A$.

THEOREM 3.1. The function $\lambda$ belongs locally to $A^{\wedge}$ at each point of $\Delta(A)$ if and only if there is an approximate identity $\left\{e_{\alpha}\right\}$ whose Gelfand transforms have compact support such that for each $\alpha, e_{\alpha}{ }^{(}(\tau) \lambda(\tau)=f_{\alpha}{ }^{\prime}(\tau)$ for some $f_{\alpha} \in A$.

Proof. By referring to the proof of Theorem 2.5 one can easily see that if $\lambda$ belongs locally to $A^{\wedge}$ then $\lambda(\tau) e_{\alpha}{ }^{\wedge}(\tau)$ belongs locally to $A^{\text {^. }}$.

Conversely, given any $\tau_{0} \in \Delta(A)$ there exists a neighborhood $V$ of $\tau_{0}$ such that $\hat{e_{\alpha_{j}}}(\tau) \neq 0$ for some $\alpha_{0}$ and all $\tau \in V$. Thus there is a $\widehat{g_{\alpha_{j}}}$ such that $g_{\alpha_{v}}(\tau)=1 / \hat{\alpha_{\alpha_{j}}}(\tau)$ for all $\tau \in V$. But there is an $f_{\alpha_{0}}$ such that $\hat{e_{\alpha_{0}}}(\tau) \lambda(\tau)=f_{\alpha_{u}}(\tau)$ for some $\tau$. Hence, for $\tau \in V$ we have $\lambda(\tau)=f_{\alpha_{\lrcorner}}(\tau) \hat{g_{\lrcorner}}(\tau)$ so that $\lambda$ belongs locally to $A^{\wedge}$.

In Corollary 2.3 necessary and sufficient conditions were given in order that $\lambda$ be the transform of a bounded multiplier. However, for specific algebras the inequality in that corollary may be hard to check. The following theorem gives another necessary and sufficient condition that $\lambda$ be the transform of a bounded multiplier.

THEOREM 3.2. In order that the function $\lambda$ on $\Delta(A)$ be the transform of a bounded multiplier it is necessary and sufficient that $\lambda \hat{e_{\alpha}}=\hat{g_{\alpha}}$ where $g_{\alpha} \in A$, and there exists $M>0$ such that $\left\|g_{\alpha}\right\| \leqq$ $M$ for all $\alpha$.

Proof. The necessity is clear. Assume therefore, that $\lambda \hat{e_{\alpha}}=\hat{g_{\alpha}}$ where $g_{\alpha} \in A$ and $\left\|g_{\alpha}\right\| \leqq M$ for all $\alpha$. Define $T(x)=y$ where $y^{\wedge}=$ $\lambda x^{\wedge}$ and $\mathscr{D}(T)=\left\{x \in A \mid \lambda x^{\wedge} \in A^{\wedge}\right\}$. It is easy to see that $\mathscr{D}(T)$ is an ideal and, since $\left\{e_{\alpha}\right\}$ is contained in $\mathscr{D}(T)$, it is dense. It follows directly from the definition that $T$ is a multiplier. Now let $f \in$ $\mathscr{D}(T)$. Then $T\left(e_{\alpha}\right) f=T\left(e_{\alpha} f\right)=T(f) e_{\alpha}$ and $T(f) e_{\alpha} \rightarrow T(f)$. Therefore $\|T(f)\| \leqq \sup _{\alpha}\left\|T\left(e_{\alpha}\right) f\right\| \leqq M\|f\|$.

\section{REFERENCES}

1. F. Birtel, On a commutative extension of a Banach algebra, Proc. Amer. Math. Soc., 13 (1962), p 818.

2. R. Larsen, An Introduction to the Theory of Multipliers, Grundlehren der mathematischen Wissenschaften, Springer-Verlag, 1969.

3. — The Multiplier Problem, Lecture Notes in Mathematics, Springer-Verlag, 1969. 
4. J. A. Wood, A necessary and sufficient condition that a function on the maximal ideal space of a Banach algebra be a multiplier, Proc. Amer. Math. Soc., 66, number 1, (1977), 39.

Received October 3, 1978 and in revised form June 5, 1979.

Virginia Commonwealth University, Richmond, VA 23284 


\section{PACIFIC JOURNAL OF MATHEMATICS}

\section{EDITORS}

DONALD BABBITT (Managing Editor)

University of California

Los Angeles, CA 90024

HUgo RossI

University of Utah

Salt Lake City, UT 84112

C. C. Moore and ANDrew OGG

University of California

Berkeley, CA 94720

\section{J. DUGUNDJI}

Department of Mathematics

University of Southern California

Los Angeles, CA 90007

R. FinN and J. MILGRaM

Stanford University

Stanford, CA 94305

\section{ASSOCIATE EDITORS}
E. F. BECKENBACH
B. H. NeumanN
F. WOLF
K. YOSHIDA

\section{SUPPORTING INSTITUTIONS}

UNIVERSITY OF BRITISH COLUMBIA CALIFORNIA INSTITUTE OF TECHNOLOGY

UNIVERSITY OF CALIFORNIA

MONTANA STATE UNIVERSITY

UNIVERSITY OF NEVADA, RENO

NEW MEXICO STATE UNIVERSITY

OREGON STATE UNIVERSITY

UNIVERSITY OF OREGON
UNIVERSITY OF SOUTHERN CALIFORNIA

STANFORD UNIVERSITY

UNIVERSITY OF HAWAII

UNIVERSITY OF TOKYO

UNIVERSITY OF UTAH

WASHINGTON STATE UNIVERSITY

UNIVERSITY OF WASHINGTON

The Supporting Institutions listed above contribute to the cost of publication of this Journal, but they are not owners or publishers and have no responsibility for its content or policies.

Mathematical papers intended for publication in the Pacific Journal of Mathematics should be in typed form or offset-reproduced, (not dittoed), double spaced with large margins. Please do not use built up fractions in the text of the manuscript. However, you may use them in the displayed equations. Underline Greek letters in red, German in green, and script in blue. The first paragraph or two must be capable of being used separately as a synopsis of the entire paper. Please propose a heading for the odd numbered pages of less than 35 characters. Manuscripts, in triplicate, may be sent to any one of the editors. Please classify according to the scheme of Math. Reviews, Index to Vol. 39. Supply name and address of author to whom proofs should be sent. All other communications should be addressed to the managing editor, or Elaine Barth, University of California, Los Angeles, California, 90024.

50 reprints to each author are provided free for each article, only if page charges have been substantially paid. Additional copies may be obtained at cost in multiples of 50 .

The Pacific Journal of Mathematics is issued monthly as of January 1966. Regular subscription rate: $\$ 84.00$ a year (6 Vols., 12 issues). Special rate: $\$ 42.00$ a year to individual members of supporting institutions.

Subscriptions, orders for numbers issued in the last three calendar years, and changes of address should be sent to Pacific Journal of Mathematics, P.O. Box 969, Carmel Valley, CA 93924, U.S.A. Older back numbers obtainable from Kraus Periodicals Co., Route 100, Millwood, NY 10546.

PUBLISHED BY PACIFIC JOURNAL OF MATHEMATICS, A NON-PROFIT CORPORATION

Printed at Kokusai Bunken Insatsusha (International Academic Printing Co., Ltd.). 8-8, 3-chome, Takadanobaba, Shinjuku-ku, Tokyo 160, Japan.

Copyright (C) 1979 by Pacific Journal of Mathematics Manufactured and first issued in Japan 


\section{Pacific Journal of Mathematics}

\section{Vol. 85, No. $2 \quad$ October, 1979}

Charles A. Akemann and Steve Wright, Compact and weakly compact derivations of $C^{*}$-algebras ........................... 253

Dwight Richard Bean, Andrzej Ehrenfeucht and George Frank McNulty, Avoidable patterns in strings of symbols ................... 261

Richard Clark Brown, Notes on generalized boundary value problems in Banach spaces. I. Adjoint and extension theory.................

Kenneth Alexander Brown and John William Lawrence, Injective hulls of group rings .................................... 323

Jacob Burbea, The Schwarzian derivative and the Poincaré metric ....... 345

Stefan Andrus Burr, On the completeness of sequences of perturbed polynomial values ....................................

Peter H. Chang, On the characterizations of the breakdown points of quasilinear wave equations..............................

Joseph Nicholas Fadyn, The projectivity of $\operatorname{Ext}(T, A)$ as a module over

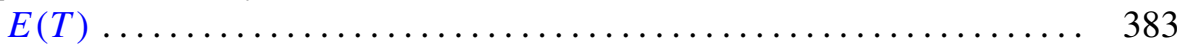

Donald Eugene Maurer, Arithmetic properties of the idèle discriminant .... 393

Stuart Rankin, Clive Reis and Gabriel Thierrin, Right subdirectly irreducible semigroups ................................. 403

David Lee Rector, Homotopy theory of rigid profinite spaces. I ........ 413 Raymond Moos Redheffer and Wolfgang V. Walter, Comparison theorems for parabolic functional inequalities................

H. M. (Hari Mohan) Srivastava, Some generalizations of Carlitz's theorem

James Alan Wood, Unbounded multipliers on commutative Banach algebras....

T. Yoshimoto, Vector-valued ergodic theorems for operators satisfying norm

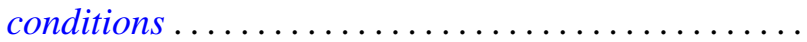

Jerry Searcy and B. Andreas Troesch, Correction to: "A cyclic inequality and a related eigenvalue problem".

Leslie Wilson, Corrections to: "Nonopenness of the set of Thom-Boardman maps" 